\title{
Populations in small, ephemeral habitat patches may drive dynamics in a Daphnia magna metapopulation
}

\author{
Florian Altermatt $^{1,2,3,4}$ And Dieter Ebert ${ }^{1,2}$ \\ ${ }^{1}$ Zoological Institute, University of Basel, Vesalgasse 1, CH-4051 Basel, Switzerland \\ ${ }^{2}$ Tvärminne Zoological Station, SF-10900 Hanko, Finland \\ ${ }^{3}$ Department of Environmental Science and Policy, University of California, Davis, California 95616 USA
}

\begin{abstract}
Migration is the key process to understand the dynamics and persistence of a metapopulation. Many metapopulation models assume a positive correlation between habitat patch size or stability and the number of emigrants. However, few empirical data exist, and habitat patch size and habitat stability may affect dispersal differently than they affect local persistence. Here, we studied the production of the migration stage (i.e., resting eggs called ephippia) of the cladoceran Daphnia magna in a metapopulation consisting of 530 rock pool habitat patches over 25 years. Earlier, the functioning of this metapopulation was explained with a Levins-type metapopulation model or with a mainland-island metapopulation model, based on local extinction and colonization data or time series data, respectively. We used pool volume, hydroperiod length, and number of desiccation events to calculate per-pool production of ephippia (i.e., migration stages). We estimated that populations in small and ephemeral habitat patches produced more than half of the 250000 to 1 million ephippia that were produced in the metapopulation as a whole per year between 1982 and 2006 . Furthermore, these small populations contributed $\sim 90 \%$ of the ephippia exposed during desiccation events, while the contribution of the long-lived populations in large pools was minimal. We term this an "inverse mainland-island" type metapopulation and propose that populations in small, ephemeral habitat patches may also be the driving force for metapopulation dynamics in other systems.
\end{abstract}

Key words: Daphnia magna; dispersal stage; ephippium; Levins metapopulation; mainland-island; metapopulation dynamics; migration.

\section{INTRODUCTION}

Many animal and plant species occur in metapopulations, consisting of spatially delimited local populations coupled by migration (Levins 1970, Hanski and Gaggiotti 2004). Metapopulation studies emphasize migration, gene flow, local extinction, and spatially correlated dynamics over and above the spatial structure of the habitat (Hanski and Gaggiotti 2004). Migration is the process that genetically connects separated populations and enables colonization of empty habitat patches, counteracting local extinction (Clobert et al. 2001, Ovaskainen and Hanski 2004). Without migration, a metapopulation would eventually go extinct. Therefore, understanding the production of migration stages, that is, their sites of origin and number, is central for the understanding of metapopulation dynamics and is the focus of this study. For instance, it may be that only patches of certain quality or size produce the majority of migrants.

In Levins' (1970) original metapopulation model, all habitat patches are equal in quality and size, and all

Manuscript received 28 October 2009; revised 27 January 2010; accepted 5 March 2010. Corresponding Editor: K. L. Cottingham

${ }^{4}$ E-mail: faltermatt@bluewin.ch populations contribute equally to metapopulation processes (Fig. 1). Also in extensions of Levins' model, each population contributes an equal number of migrants (Hanski 1999). Later models consider migration as a function of the patch size, or population size or density in the patch of origin (Travis et al. 1999), and the number of emigrants is assumed to be higher in larger populations or at higher population densities. Eventually, in mainland-island metapopulations (Fig. 1; Boorman and Levitt 1973, Harrison 1991) and also sourcesink metapopulations (Brown and Kodric-Brown 1977, Kawecki 2004), habitat persistence is considered. In a mainland-island metapopulation, a few large populations in usually large "mainland" patches have a negligible risk of extinction, while small populations in small "island" patches have a high risk of extinction (Harrison 1991). Implicitly, the mainland-island model makes a statement about the origin of migrants, as colonization of empty patches is enhanced by migration from the mainland (Hanski 1999). Overall, metapopulation dynamics are driven by the migrants coming from the few permanent mainland populations, while island populations are negligible (Harrison 1991, Hanski 1999, Kawecki 2004). The source-sink model focuses on demography rather than on local population size (Brown and Kodric-Brown 1977, Kawecki 2004). 


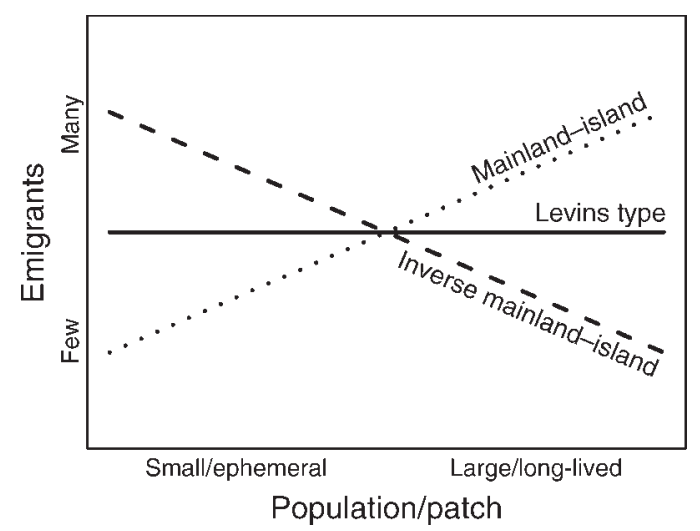

FIG. 1. Schematic depiction of a population's contribution of emigrants relative to the persistence or size of the population. In a Levins metapopulation all habitat patches are equal in quality and size. Metapopulations with different-sized habitat patches may still figuratively fit into that category when populations contribute equal number of migrants, irrespective of the patch size (straight line). In a mainland-island metapopulation (dotted line), emigrants are mostly originating from a few large, long-lived populations. In an "inverse mainland-island" metapopulation (dashed line), migrants are mostly originating from small, ephemeral populations.

Reproduction in sink populations does not balance mortality, and sink populations are only maintained by immigration from source populations. Again, populations in large habitat patches are implicitly seen as source populations (Kawecki 2004). The intention of these models is to make more nuanced inferences about how natural metapopulations function (Harrison 1991).

Local extinction has been a central issue of many empirical studies (reviewed in Hanski and Gaggiotti 2004), but the equally important question of colonization (as a balance for local extinction) has been much less studied (Clobert et al. 2001, Hanski and Gaggiotti 2004). Relatively few empirical studies have assessed the origin and number of migrants within an entire metapopulation (reviewed in Clobert et al. 2001, Bullock et al. 2002). Hanski et al. (2000) used mark-recapture data from a Melitaea diamina butterfly metapopulation to estimate rates of migration among populations and scale them with habitat patch area and isolation. In their model, emigration was proportional to patch area, and populations in large patches contribute more migrants than populations in small patches. Likewise, in the Glanville fritillary (Melitaea cinxia) metapopulation on the Aland Islands, the number of migrants was estimated by monitoring the occupation of patches as well as mark-recapture measurements (reviewed in Ehrlich and Hanski 2004). Again, large and long-lasting populations contributed most of the migrants. However, population size may not be the best criterion to identify populations that are key to metapopulation dynamics. For example, Crone et al. (2001) found that the stable long-term equilibrium in a vole metapopulation was only maintained by the small and ephemeral subpopu- lations that promote emigration. Since the common assumption, that habitat patch size and habitat stability affect dispersal in the same way as they affect local persistence, may not be generally true, a focus on the origin of migrants may be especially important for a better understanding of natural metapopulations.

To address the relationship between the habitat patch of origin and number of migrants, we estimated the production of the migration stage (i.e., resting eggs called ephippia) in a well-studied Daphnia magna rock pool metapopulation (Hanski and Ranta 1983, Pajunen 1986, Ebert et al. 2001, Pajunen and Pajunen 2003). We were especially interested in the production of ephippia in relation to pool volume and habitat stability as well as in the overall production of ephippia in the whole metapopulation. We were motivated by two different opinions on how this metapopulation functions. Based on local extinction and colonization data, the processes in this metapopulation were either explained with a typical Levins-type metapopulation model (Hanski and Ranta 1983, Hugueny et al. 2007), or, based on time series data, with a mainland-island metapopulation model (Pajunen 1986, Pajunen and Pajunen 2003). However, both assessments were made without data on the production of ephippia and their exposure to migration. Here we closed this gap and came up with a very different explanation to explain the metapopulation processes.

Our study considered populations in 530 potential habitat patches (rock pools) over 25 years. Here, we estimate annual ephippium production at the metapopulation level. We were not only interested in the overall production of ephippia, but also in the contribution of populations in small habitat patches, and how often ephippia in small pools get exposed to passive dispersal by wind and birds. Based on these estimates we propose that populations in small, ephemeral habitat patches contributed most to the total quantity of migrants and may drive the dynamics in this Daphnia magna metapopulation. We term this ecological setting an "inverse mainland-island metapopulation" (Fig. 1), and argue why these results may be more general and not specific only to the Daphnia system.

\section{Material and Methods}

\section{The natural system}

The freshwater crustacean Daphnia magna Straus, 1820 (Crustacea: Cladocera) is widely distributed along the coast of the Baltic Sea. It inhabits rock pools ranging in volume from $\sim 10$ to $50000 \mathrm{~L}$ on the skerry islands of southwest Finland (Pajunen and Pajunen 2003). An average D. magna population persists for about 3-5 years (Pajunen and Pajunen 2003). Pajunen and Pajunen (Pajunen 1986, Pajunen and Pajunen 2003) defined longlived populations in large pools (having a low risk of desiccation) as mainland populations. Daphnia hatch from ephippia at the beginning of May, and the populations are in their planktonic phase until about 
the end of September. During this time, D. magna reproduces predominantly asexually, interrupted by periods of sexual reproduction when ephippia are produced. As in other aquatic organisms with resting stages, ephippia also serve as wind-drifted dispersal stages (Maguire 1963, Vanschoenwinkel et al. 2008). Most rock pools contain very little sediment, and, contrary to lakes, do not have yearly strata of sediment. In our terminology, a Daphnia population consists of both the planktonic animals and the ephippia.

\section{Data collection}

For the current study we combined published data from long-term metapopulation monitoring (Pajunen and Pajunen 2003) with a hydrological model (Altermatt et al. 2009) and estimates on the production of ephippia in individual populations (Altermatt and Ebert 2008).

The D. magna metapopulation data set was collected by Pajunen and Pajunen (2003) and includes all rock pools from 18 neighboring islands. The islands are part of a much larger and possibly continuous metapopulation, comprising thousands of islands all along the coast of the Baltic Sea (Bengtsson 1989). To simplify matters, when using the term "metapopulation" we only refer to the pools in our study area, and not to all pools along the Baltic coast. Pajunen and Pajunen (2003) have monitored 530 rock pools on these islands for the presence or absence of D. magna over 25 years (19822006; for details see Pajunen [1986] and Pajunen and Pajunen [2003]). Data on the longest axis of each pool, the greatest width perpendicular to this axis, and the maximal water depth were available from all pools, either collected by us or Pajunen (1986). Following the method of Ebert et al. (2001), we estimated the volume of each rock pool as an inverted pyramid ([width $\times$ length $\times$ depth]/3).

A hydrological model was developed to predict poolspecific evaporation rates, and eventual desiccation (for details see Altermatt et al. 2009). In short, pool-specific variables (pool surface and presence or absence of vegetation), and environmental variables (standardized evaporation, daily temperature, wind, and season) were used to predict daily evaporation in each pool. Desiccation was predicted by using local precipitation data and the pool-specific catchment area (Altermatt et al. 2009), the inflow of water, and by knowing pool depth. Based on daily water level estimates, the model predicts hydroperiod length (in days) and the number of desiccation events for each pool for every year from 1982 to 2006.

Finally, we used the linear regressions given by Altermatt and Ebert (2008) to estimate the production of ephippia in individual populations. Altermatt and Ebert (2008) measured the daily production of ephippia relative to pool volume and mesocosm volume, respectively. Here, we used the mean slope $(0.215)$ and mean intercept (1.096) of these regression models to predict the production of ephippia in pools of any volume (both axes were on a $\log _{10}$ scale).

\section{Calculation of the production and exposure of ephippia}

We used probability density estimates to describe the distribution of populations in pools of different volume over 25 years from 1982 to 2006. Probability density estimates are mathematical models that give continuous estimates of a probability distribution based on observed data (Venables and Ripley 2002), in our case, pool volume. Kernel density estimates (Venables and Ripley 2002) were used to get the continuous probability density distribution of the yearly inhabited pools (Fig. 2A). These estimates can be seen as "smoothed" versions of histograms, where the area under the curve adds up to one (or 100\%). Multiplying the probability density distribution with the total number of observed data gives the frequency distribution with absolute values on the $y$-axis, and the area under the curve matches the number of observed data. The continuous estimates over all pool volumes are the main advantage of the probability density method compared to discontinuous methods such as histograms.

We considered the annual time period between 1 May and 30 September, which is relevant for the planktonic phase of Daphnia (Altermatt and Ebert 2008). We multiplied the estimates on the daily production of ephippia (Altermatt and Ebert 2008) with the yearly frequency distributions of occupied pools over the range of volumes (as shown in Fig. 2A). This resulted in a continuous curve with the ephippia produced per day for all occupied rock pools in our metapopulation in any given year (Fig. 2B). In a next step we extended the estimates of ephippium production per day to total annual production. Ephippia are only produced when pools contain water, and we therefore adjusted the production for the hydroperiod length, thus excluding the periods when pools were dry (Altermatt and Ebert 2008). Using the existing evaporation model (Altermatt et al. 2009), we predicted the yearly hydroperiod length relative to the volume for all pools that contained a $D$. magna population. We modeled hydroperiod length (in days) and desiccation events for all pools separately (Altermatt et al. 2009). We used simple nonlinear least square fits to get a continuous predictor of annual hydroperiod length relative to pool volume (see Appendix: Figs. A1, A2). These curves give the hydroperiod length relative to pool volume. We used these estimates to correct for different hydroperiod lengths in pools of different volume, when extending the daily production of ephippia (Fig. 2B) to the annual production, (Fig. $2 \mathrm{C}$ ) by multiplying the value from Fig. $2 \mathrm{~B}$ with the corresponding yearly curve from Appendix: Fig. A1 over the range of all pool volumes.

Finally, we predicted the contribution to the yearly total production and exposure of ephippia. We assumed that ephippia get exposed to migration at each desiccation event (Vanschoenwinkel et al. 2008). We 


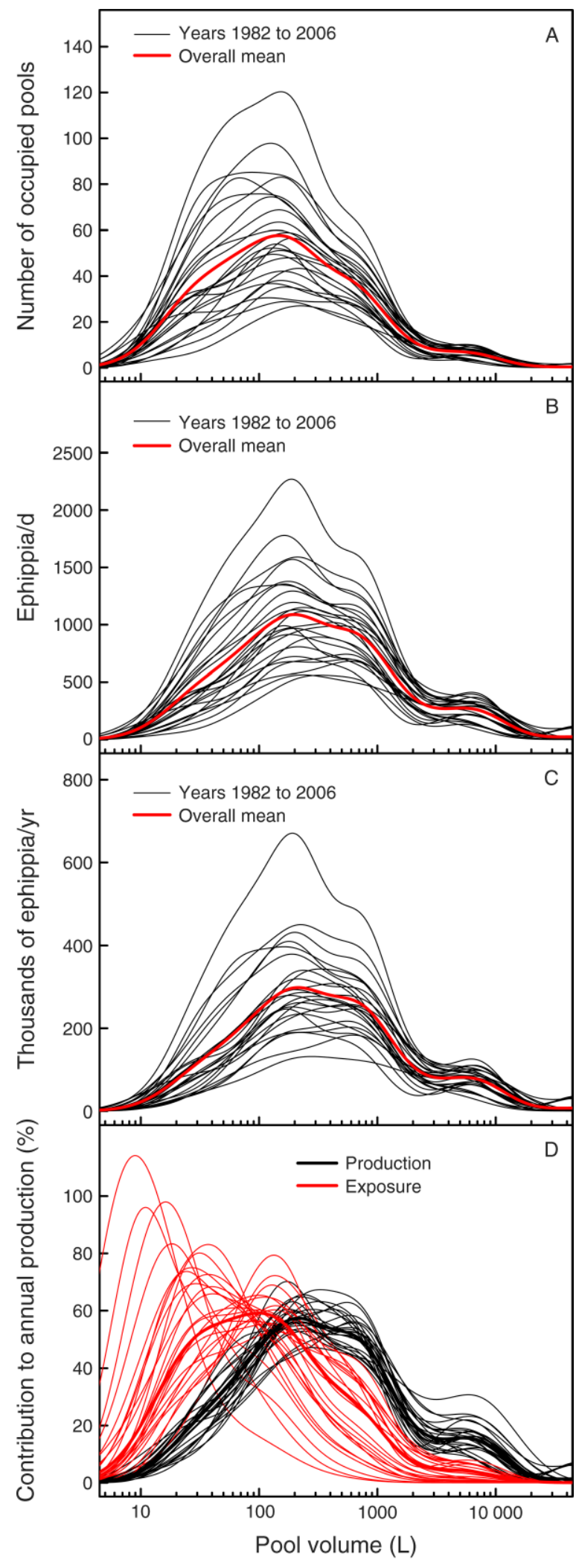

compared pool volume and predicted number of desiccation events with nonlinear least squares models to get a yearly continuous predictor of desiccation events relative to pool volume (Appendix: Fig. A2).

\section{Statistical analysis}

Statistical analyses were performed with R ( R Development Core Team 2008), version 2.10.1, and also using the software package "Date." Probability density estimates were conducted according to Venables and Ripley (2002). We used $\log _{10}$-transformed pool volume data, the default kernel, and default bandwidth functions implemented in $\mathrm{R}$. We used nonlinear least square fits to relate the yearly predicted time a pool was dry (period 1 May to 30 September) with its volume. These predictions were used to scale the percentage of time when ephippia could be produced relative to pool volume. We used nonlinear least square fits to relate the number of desiccation events per year with pool volume. Again, these predictions were used to scale the contribution of exposed ephippia. We calculated the number of colonizations and colonization odds in the $D$. magna metapopulation from the long-term monitoring data (Pajunen and Pajunen 2003, Altermatt et al. 2008), and used the colonization odds as a measure of the overall migration rate in the metapopulation. We used generalized linear models with a binomial error distribution to compare colonization odds with the production and exposure of ephippia (Fig. 3).

\section{Results \\ Rock pool volume}

The volume of the 530 rock pools was 4-48000 L. Daphnia magna was found in pools of practically all sizes. The size of pools that were ever inhabited was $6-$ 48000 L. About $80 \%$ of the populations occurred in pools with a volume of 20-600 L (Fig. 2A). The peak of the probability density estimates of all occupied pools was in $120 \mathrm{~L}$ volume pools (Fig. 2A). The number of

FIG. 2. Frequency distributions on occupancy and ephippium production in relation to pool volume. (A) Estimated absolute frequency distributions of all rock pools that were occupied by D. magna (see Material and methods). Yearly values from 1982 to 2006 (thin lines) and the overall mean (thick red line) are shown. (B) Estimated absolute frequency distribution of the daily ephippium production in the whole metapopulation. For each specific year, the daily production of ephippia was calculated relative to pool volume and the absolute frequency distribution of occupied pools. (C) Estimated absolute frequency distribution of the total production of ephippia in the metapopulation per year. The production of ephippia was calculated by multiplying the daily estimates from panel (B), but correcting for hydroperiod length. (D) Predicted percentage contribution to the yearly total production (black lines) and exposure (red lines) of ephippia in the whole D. magna metapopulation relative to pool volume (thin lines represent yearly values; the thick line represents the overall mean). 
occupied pools varied markedly between years (Fig. 2A; area under the curve), and so did the occupation length in different pools (data not shown, but see Pajunen and Pajunen 2003).

\section{Ephippium production}

Estimates on the average number of ephippia produced per day in the whole metapopulation for each year from 1982 to 2006 are given in Fig. 2B. Considering the average daily production, most ephippia were produced by populations in pools of $20-1000 \mathrm{~L}$ (Fig. 2B). However, these estimates did not consider the different hydroperiod lengths for pools of different volumes. Consistent with our expectations, hydroperiod length was associated with pool volume and shortened when pool volume decreased (Appendix: Fig. A2; see also Altermatt et al. 2009). However, there was considerable year-to-year variation in the slope of the nonlinear least square fits between the predicted hydroperiod length and pool volume (Appendix: Fig. A1).

The estimates on the yearly production of ephippia within the whole metapopulation ranged from 250000 to 1060000 ephippia for the years 1982-2006 (mean 510000 ephippia, median 496000 ephippia; Fig. 2C). The majority $(\sim 60 \%)$ of the ephippia were produced by populations in pools of $100-1000$ L (Fig. 2C). Only about $20 \%$ of all ephippia were produced by the populations in the largest pools $(>1000 \mathrm{~L})$.

Finally, we estimated the export of ephippia through passive dispersal by wind and active dispersal by birds. The nonlinear least squares fits between the number of desiccation events and pool volume showed that pools $>20$ L may experience up to four desiccations per year, and that desiccation depended on pool volume and year (Appendix: Fig. A2). Using these estimates of desiccation events, we then calculated the percentage contribution to the production and exposure of ephippia by populations in pools of different volume and years (Fig. 2D). We found that almost $90 \%$ of all exposed ephippia originated from pools that were $<300 \mathrm{~L}$ (Fig. 2D), even though only $\sim 50 \%$ of all ephippia were produced by these populations. The remaining $10 \%$ of exposed ephippia originated from populations in pools of 300$1000 \mathrm{~L}$. Large pools (>1000 L) barely contributed to the exposed ephippia.

\section{Correlations between ephippium production and colonization rate}

If ephippium exposure to wind and birds contributes to dispersal, we expected to find a correlation between the estimated number of exposed ephippia and the number of newly colonized pools in the following year (after diapause). We did indeed find a significant correlation between the predicted number of ephippia exposed to migration (during desiccation events) in a given year and the colonization odds in the subsequent year (generalized linear model, $Z=5.83, P<0.0001$; Fig. 3A). In contrast, we did not find a significant

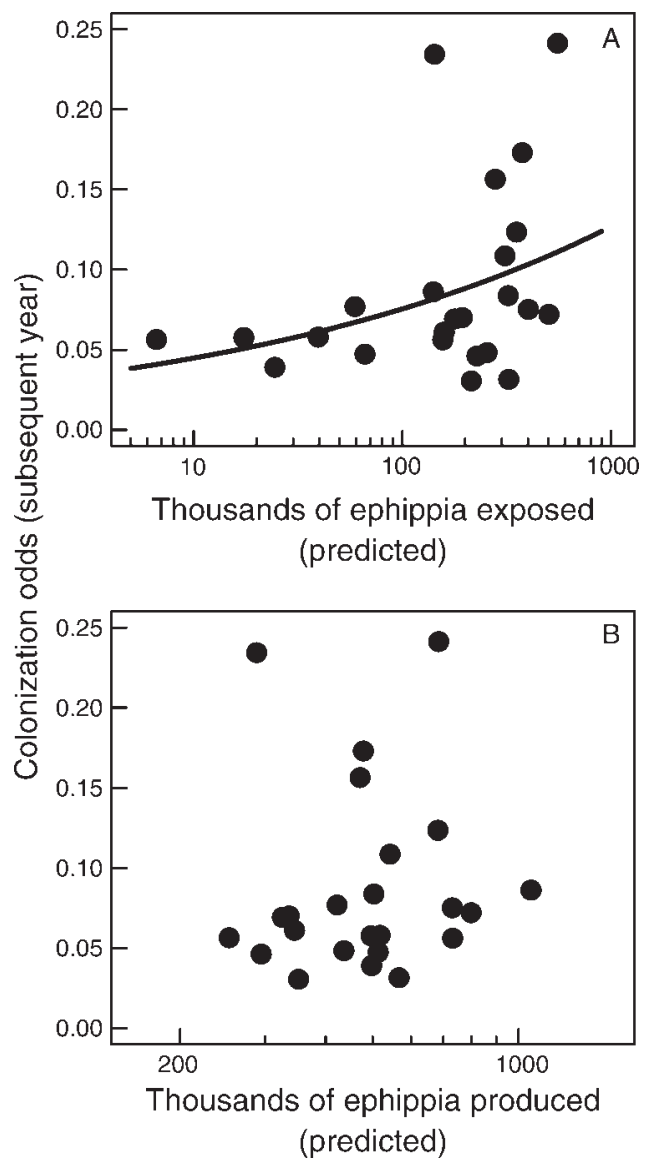

Fig. 3. (A) Colonization odds relative to the predicted number of ephippia exposed. Exposure was proportional to the number of pool-specific desiccation events. Colonization odds were significantly higher after years when a large number of ephippia were exposed (generalized linear model, $P<0.0001$ ). The fitted line gives the estimate from the generalized linear model. (B) Colonization odds relative to the predicted number of ephippia produced. There was no significant relationship between the total production of ephippia and the colonization odds in the subsequent season (generalized linear model; $P=$ 0.22 ). Note logarithmic scales on the $x$-axes.

correlation between the predicted total number of ephippia produced (independent of exposure) in any given year and the colonization odds in the subsequent year (generalized linear model, $Z=1.22, P=0.22$; Fig. 3B).

\section{Discussion}

The long-term persistence of a metapopulation depends on colonization balancing local extinction (Levins 1970). Thus, to understand and protect metapopulations it is essential to identify the populations from which most migrants originate. In the past, the understanding of the metapopulation was mostly based on colonization-extinction dynamics (Bengtsson 1989, Harrison 1991, Hanski and Gaggiotti 2004), and the main focus was put on which populations go extinct, and less on where the migrants that colonize new habitat 
patches come from. Here, we show that the conclusions about the functioning of a metapopulation may be different when the origin and number of migrants is considered, rather than using extinction-colonization patterns alone. We present evidence for a Daphnia magna metapopulation in which populations in small and ephemeral habitat patches apparently contribute the most to production of migration stages and may drive metapopulation dynamics (Figs. 2 and 3). This metapopulation may thus neither be explained by the original Levins-type metapopulation model (Levins 1970) (Fig. 1), nor by later models (Hanski 1999, Travis et al. 1999). We term our metapopulation an "inverse mainlandisland metapopulation." This term is analogous to the classic mainland-island type metapopulation (Fig. 1; Boorman and Levitt 1973, Harrison 1991), in which, however, the few large and long-lived populations are the driving force for metapopulation processes.

Our argument based on our findings has two key aspects. First, populations in small and ephemeral habitat patches are numerically predominant. Second, these populations produce proportionally more migrants than large and long-lived populations in large habitat patches. These factors can work separately or in combination. They may be valid for metapopulations of other organisms as well, and the herein-described inferences about the functioning of a metapopulation and the significance of populations in small and ephemeral habitat patches may be more general.

Daphnia has been a classic study organism for many questions in metapopulation biology (Hanski and Ranta 1983, Pajunen 1986, Bengtsson 1989, Ebert et al. 2001, Pajunen and Pajunen 2003, Hugueny et al. 2007, Altermatt et al. 2008). Surprisingly, the numerical predominance of populations in small and ephemeral habitat patches and the disproportionately large production of ephippia in these populations has not been studied and adequately acknowledged. Also, environmental conditions in small pools are more variable, which triggers ephippium production for Daphnia (Carvalho and Hughes 1983), and may be a mechanistic explanation for the numerically large production of ephippia. We postulate that when species' traits affecting local survival are negatively correlated with traits affecting the production of migrants, metapopulations may have "inverse mainland-island" dynamics. In our study, we used model-based estimates of numbers of ephippia, rather than actual counts of ephippia. While this might have introduced some uncertainty, we were thereby able to draw conclusions on a large number of populations and over a long period of time. Also, in many other organisms, the production of migrants and the tendency to migrate and disperse is most pronounced in temporary habitats and less in stable habitats (already reviewed by Southwood 1962). Examples are aquatic rock pool arthropods such as Daphnia longispina and D. pulex, copepods, and ostracods, which locally co-occur with $D$. magna and have similar life cycles and dispersal stages (Pajunen 1986). Also, recent data show that migrants in butterfly metapopulations are not a random subset of all populations. Specific genotypes, related to newly established populations, are more likely to disperse (Hanski and Saccheri 2006), and dispersal may be an inversely density-dependent behavior (Ehrlich and Hanski 2004), promoting dispersal from small or marginal populations. In a study on fragmented forest patches in Madagascar, Bodin et al. (2006) found that ecosystem services provided by forest patches may be well maintained irrespective of the size of the patches, and mostly depend on their spatial distribution. Even in mammal metapopulations, migrants may come predominantly from ephemeral subpopulations (Crone et al. 2001) and significantly contribute to metapopulation persistence. Crone et al. (2001) found that vole densities in a natural metapopulation were not only less stable on smaller islands, but also that emigration increased before the extinction of a subpopulation.

In our study system, the numerical production of ephippia is only part of the story, since dispersal of ephippia is passive (Maguire 1963) and occurs predominantly when pools are dry (Vanschoenwinkel et al. 2008). When incorporating the exposure of ephippia into our model, we found a remarkable shift in the contribution of exposed ephippia toward pools of the smallest size classes (Fig. 2D). We validated the idea that migrants originated from dry pools by using our predictions of the number of produced and exposed ephippia, and comparing them with observed dispersal rates. Consistent with other studies (as shown by Vanschoenwinkel et al. 2008), we found significantly higher colonization rates after years in which we estimated a numerically large exposure of ephippia in the metapopulation by desiccation (Fig. 3A). On the contrary, when exposure was not considered, we did not find such a correlation (Fig. 3B). The absence of a positive correlation in the latter case suggests that migration does not directly follow a mass effect of the number of ephippia that were produced, but rather depends on desiccation. For the metapopulation studied herein, desiccation is not a detrimental form of disturbance, but is positively associated with dispersal (Kneitel and Chase 2004) and eventually promotes metapopulation persistence. Conversely, populations in large pools may not contribute much to the overall metapopulation dynamics. The ephippia produced in these large pools may only guarantee local long-term survival of populations (Pajunen and Pajunen 2003), which was used as an argument for a mainland-island metapopulation (Pajunen 1986). Eventually, however, populations go extinct even in large and permanent pools, and the largest pools did not contain permanent populations, possibly because of the occasional occurrence of fish or the accumulation of parasites over time (Pajunen 1986, Ebert et al. 2001, Pajunen and Pajunen 2003). Since extinction is a common event (Pajunen 1986, Pajunen and Pajunen 2003), the long-term persistence of the metapopulation is only 
possible when migration and subsequent colonization is equally common.

Parasites may be an additional factor that gives migrants from populations in small and ephemeral pools an advantage. Generally, both parasite richness as well as parasite prevalence increases with host population age (Ebert et al. 2001), and it is mostly the short-lived populations that are free of parasites. Since uninfected Daphnia have a much higher immigration success than infected Daphnia (Altermatt et al. 2007), the mostly uninfected migrants from short-lived populations have an additional advantage compared to possibly infected migrants from long-lived and highly parasitized old populations. Even when short-lived populations in ephemeral habitats may not be the exclusive source of migrants, both desiccation as well as parasitism strongly favors migration from these populations.

Even though D. magna is not a species of conservation concern, the identification of the key populations within a metapopulation may be an essential issue in conservation biology, since many metapopulations are challenged by habitat fragmentation and global climate change (Clobert et al. 2004, Altermatt et al. 2008). Conservation efforts are often limited, and may be directed only to a subset of all populations (ArroyoRodríguez et al. 2009). In many cases, the large and long-lived populations are protected, while small or ephemeral populations are neglected (Arroyo-Rodríguez et al. 2009). Our example indicates that a biased assignment of conservation efforts to large and longlived populations could sometimes interrupt metapopulation processes. In our case, the protection of a few large populations may guarantee local survival of the species, but only the protection of the small, ephemeral populations may maintain colonization of vacant habitat patches and metapopulation processes in general. In the context of a changing environment (Clobert et al. 2004, Altermatt et al. 2008), the maintenance of metapopulation processes may be essential to colonize new habitats and meet the need for range shifts.

To our knowledge, our study is one of very few examples that quantified migration stages in a metapopulation, even over many years. We found that most migration stages were produced in populations occurring in small and ephemeral habitat patches, and propose that these populations are the driving force of metapopulation dynamics, creating an "inverse mainland-island" situation (Fig. 1). Our findings may not be restricted to the Daphnia system, and could be more general, but so far overlooked, since mostly extinctioncolonization dynamics were studied and not the actual origin and number of migrants. Rock pools are a widespread and important aquatic habitat type, and many organisms occurring in rock pools (Ranta 1982) have a similar life cycle, such as ostracods, copepods, or other Cladocera. Thus, some or even all of these organisms may share similar dynamics. The dynamics described herein may also be relevant for organisms in which the resting stage may serve as a dispersal stage (i.e., most plants, in which seeds are also often wind dispersed). Further candidates are organisms in which environmental factors negatively affect local survival, but may be beneficial for migration. Our findings help to make more nuanced inferences about the functioning of natural metapopulations. The underlying mechanism is not a discrete one (as simplified in Fig. 1), but is at least in part based on quantitative differences (patch size distribution and relative contribution of migrants). We suggest that empirical studies of metapopulations should not only focus on local extinction but also on the origin and number of migrants. Future metapopulation models may take patch size distributions (or other patch attributes) and production of migrants into account, independently of extinction probability, to form a more general framework for understanding the dynamics in natural metapopulations.

\section{ACKNOWLEDGMENTS}

The study was supported by the Swiss National Science Foundation. We thank I. Pajunen for the Daphnia data set and discussion. F. Altermatt thanks the Basler Stiftung für experimentelle Zoologie, the Freiwillige Akademische Gesellschaft, and the Emilia Guggenheim-Schnurr-Stiftung for financial support during the fieldwork. We thank A. Bieger, H. Cornell, S. Harrison, M. Holyoak, I. Pajunen, W. Weisser, T. Zumbrunn, and two anonymous reviewers for comments on the manuscript. This work is part of project no. 97524006 at Tvärminne Zoological Station.

\section{Literature Cited}

Altermatt, F., and D. Ebert. 2008. The influence of pool volume and summer desiccation on the production of the resting and dispersal stage in a Daphnia metapopulation. Oecologia 157: 441-452.

Altermatt, F., J. Hottinger, and D. Ebert. 2007. Parasites promote host gene flow in a metapopulation. Evolutionary Ecology 21:561-575.

Altermatt, F., V. I. Pajunen, and D. Ebert. 2008. Climate change affects colonisation dynamics in a metacommunity of three Daphnia species. Global Change Biology 14:1209-1220.

Altermatt, F., I. Pajunen, and D. Ebert. 2009. Desiccation of rock pool habitats and its influence on population persistence in a Daphnia metacommunity. PLoS ONE 4:e4703.

Arroyo-Rodríguez, V., E. Pineda, E. Escobar, and J. BenítezMalvido. 2009. Value of small patches in the conservation of plant-species diversity in highly fragmented rainforest. Conservation Biology 23:729-739.

Bengtsson, J. 1989. Interspecific competition increases local extinction rate in a metapopulation system. Nature 340:713715 .

Bodin, O., M. Tengo, A. Norman, J. Lundberg, and T. Elmqvist. 2006. The value of small size: Loss of forest patches and ecological thresholds in southern Madagascar. Ecological Applications 16:440-451.

Boorman, S. A., and P. R. Levitt. 1973. Group selection on the boundary of a stable population. Theoretical Population Biology 4:84-128.

Brown, J. H., and A. Kodric-Brown. 1977. Turnover rates in insular biogeography: effect of immigration on extinction. Ecology 58:445-449.

Bullock, J. M., R. E. Kenward, and R. S. Hails, editors. 2002. Dispersal ecology. British Ecological Society, Blackwell, Oxford, UK. 
Carvalho, G. R., and R. N. Hughes. 1983. The effect of food availability, female culture-density and photoperiod on ephippia production in Daphnia magna Strauss (Crustacea: Cladocera). Freshwater Biology 13:37-46.

Clobert, J., E. Danchin, A. A. Dhondt, and J. D. Nichols, editors. 2001. Dispersal. Oxford University Press, Oxford, UK.

Clobert, J., R. A. Ims, and F. Rousset. 2004. Causes, mechanisms and consequences of dispersal. Pages 307-335 in I. Hanski and O. E. Gaggiotti, editors. Ecology, genetics, and evolution of metapopulations. Elsevier Academic Press, Amsterdam, The Netherlands.

Crone, E. E., D. Doak, and J. Pokki. 2001. Ecological influences on the dynamics of a field vole metapopulation. Ecology 82:831-843.

Ebert, D., J. W. Hottinger, and V. I. Pajunen. 2001. Temporal and spatial dynamics of parasites in a Daphnia metapopulation: Which factors explain parasite richness? Ecology 82: 3417-3434.

Ehrlich, P. R., and I. Hanski. 2004. On the wings of checkerspots. Oxford University Press, Oxford, UK.

Hanski, I. 1999. Metapopulation ecology. Oxford University Press, Oxford, UK.

Hanski, I., J. Alho, and A. Moilanen. 2000. Estimating the parameters of survival and migration of individuals in metapopulations. Ecology 81:239-251.

Hanski, I., and O. E. Gaggiotti, editors. 2004. Ecology, genetics and evolution of metapopulations. Elsevier Academic Press, Amsterdam, The Netherlands.

Hanski, I., and E. Ranta. 1983. Coexistence in a patchy environment: three species of Daphnia in rock pools. Journal of Animal Ecology 52:263-279.

Hanski, I., and I. J. Saccheri. 2006. Molecular-level variation affects population growth in a butterfly metapopulation. PLoS Biology 4:719-726.

Harrison, S. 1991. Local extinction in a metapopulation context: an empirical evaluation. Biological Journal of the Linnean Society 42:73-88.

Hugueny, B., H. V. Cornell, and S. Harrison. 2007. Metacommunity models predict the local-regional species richness relationship in a natural system. Ecology 88:1696-1706.
Kawecki, T. J. 2004. Ecological and evolutionary consequences of source-sink population dynamics. Pages 387-414 in I. Hanski and O. E. Gaggiotti, editors. Ecology, genetics, and evolution of metapopulations. Elsevier Academic Press, Amsterdam, The Netherlands.

Kneitel, J. M., and J. M. Chase. 2004. Disturbance, predator, and resource interactions alter container community composition. Ecology 85:2088-2093.

Levins, R. 1970. Extinction. Lecture Notes in Mathematics 2: 75-107.

Maguire, B. 1963. The passive dispersal of small aquatic organisms and their colonization of isolated bodies of water. Ecological Monographs 33:161-185.

Ovaskainen, O., and I. Hanski. 2004. Metapopulation dynamics in highly fragmented landscapes. Pages $73-103$ in I. Hanski and O. E. Gaggiotti, editors. Ecology, genetics, and evolution of metapopulations. Elsevier Academic Press, Amsterdam, The Netherlands.

Pajunen, V. I. 1986. Distributional dynamics of Daphnia species in a rock-pool environment. Annales Zoologici Fennici 23: 131-140.

Pajunen, V. I., and I. Pajunen. 2003. Long-term dynamics in rock pool Daphnia metapopulations. Ecography 26:731-738.

R Development Core Team. 2008. R: A language and environment for statistical computing. Version 2.8.0. R Foundation for Statistical Computing, Vienna, Austria.

Ranta, E. 1982. Animal communities in rockpools. Annales Zoologici Fennici 19:337-347.

Southwood, T. R. E. 1962. Migration of terrestrial arthropods in relation to habitat. Biological Reviews 37:171-211.

Travis, J. M. J., D. J. Murrell, and C. Dytham. 1999. The evolution of density-dependent dispersal. Proceedings of the Royal Society B 266:1837-1842.

Vanschoenwinkel, B., S. Gielen, M. Seaman, and L. Brendonck. 2008. Any way the wind blows - frequent wind dispersal drives species sorting in ephemeral aquatic communities. Oikos 117:125-134.

Venables, W. N., and B. D. Ripley. 2002. Modern applied statistics with S. 4th edition. Springer, New York, New York, USA.

\section{APPENDIX}

Figures showing predicted annual hydroperiod length and desiccation events relative to pool volume (Ecological Archives E091209-A1). 